ANNALES

POLONICI MATHEMATICI

$93.3(2008)$

\title{
Lifting distributions to the cotangent bundle
}

\author{
by WŁodzimierz M. Mikulski (Kraków)
}

\begin{abstract}
A classification of all $\mathcal{M} f_{m}$-natural operators $A: \operatorname{Gr}_{p} \rightsquigarrow \operatorname{Gr}_{q} T^{*}$ lifting $p$-dimensional distributions $D \subset T M$ on $m$-manifolds $M$ to $q$-dimensional distributions $A(D) \subset T T^{*} M$ on the cotangent bundle $T^{*} M$ is given.
\end{abstract}

1. A $p$-dimensional distribution on a manifold $M$ is a smooth $\left(C^{\infty}\right)$ vector subbundle $D \subset T M$ of the tangent bundle of $M$ such that $\operatorname{dim}\left(D_{x}\right)=p$ for any point $x \in M$. Thus a $p$-dimensional distribution $D$ is a smooth $\left(C^{\infty}\right)$ section of the Grassmann bundle $\mathrm{Gr}_{p} M$ of $M$.

Given a manifold $M$, let $T^{*} M=(T M)^{*}$ be the cotangent bundle of $M$. Every embedding $f: M \rightarrow N$ of $m$-manifolds induces a vector bundle embedding $T^{*} f:=\left(T\left(f^{-1}\right)\right)^{*}: T^{*} M \rightarrow T^{*} N$ covering $f$, where $T f$ denotes the differential of $f$.

A general concept of natural operators can be found in [1]. We need the following partial definition of natural operators only.

Let $m, p$ and $q$ be integers such that $m \geq 1,0 \leq p \leq m$ and $0 \leq$ $q \leq 2 m$. An $\mathcal{M} f_{m}$-natural operator $A: \mathrm{Gr}_{p} \rightsquigarrow \mathrm{Gr}_{q} T^{*}$ lifting $p$-dimensional distributions $D$ from $m$-manifolds $M$ to $q$-dimensional distributions $A(D)$ on the cotangent bundle $T^{*} M$ is a family of $\mathcal{M} f_{m}$-invariant regular operators (functions)

$$
A: \underline{\operatorname{Gr}_{p}}(M) \rightarrow \underline{\operatorname{Gr}_{q}}\left(T^{*} M\right)
$$

from the set $\operatorname{Gr}_{p}(M)$ of all $p$-dimensional distributions on $M$ (sections of $\left.\operatorname{Gr}_{p} M \rightarrow M\right)$ into the set $\operatorname{Gr}_{q}\left(T^{*} M\right)$ of all $q$-dimensional distributions on $T^{*} M$ (sections of $\left.\operatorname{Gr}_{q}\left(T^{*} M\right) \rightarrow T^{*} M\right)$ for any $\mathcal{M} f_{m}$-object $M$, where $\mathcal{M} f_{m}$ is the category of $m$-dimensional manifolds and their embeddings. The $i n$ variance means that if $D_{1} \in \operatorname{Gr}_{p}(M)$ and $D_{2} \in \operatorname{Gr}_{p}(N)$ are $f$-related (i.e. $\left.\operatorname{Gr}_{p} f \circ D_{1}=D_{2} \circ f\right)$ for some $\mathcal{M} f_{m}$-map $f: \bar{M} \rightarrow N$ then $A\left(D_{1}\right)$ and $A\left(D_{2}\right)$ are $T^{*} f$-related. (We recall that $\operatorname{Gr}_{p} f: \operatorname{Gr}_{p} M \rightarrow \operatorname{Gr}_{p} N$ is the map induced by $f$ given by $\operatorname{Gr}_{p} f(W)=T f(W)$ for any $W \in\left(\operatorname{Gr}_{p} M\right)_{x}$, i.e. for

2000 Mathematics Subject Classification: 58A20, 58A32.

Key words and phrases: distribution, Grassmann bundle, natural operator. 
any $p$-dimensional subspace $W \subset T_{x} M, x \in M$.) The regularity means that $A$ transforms smoothly parametrized families of distributions into smoothly parametrized families of distributions.

We have the following $\mathcal{M} f_{m}$-natural operators $A: \operatorname{Gr}_{p} \rightsquigarrow \mathrm{Gr}_{q} T^{*}$.

Example 1. $A^{[1]}(D)_{\omega}=\{0\}$ for all $\omega \in\left(T^{*} M\right)_{x}, x \in M, D \in \underline{\operatorname{Gr}_{p}}(M)$.

ExAmple 2. $A^{[2]}(D)_{\omega}=\left\{\frac{d}{d t}(\omega+t \sigma)_{t=0} \in T_{\omega} T^{*} M: \sigma \in \operatorname{Ann}\left(D_{x}\right)\right\}$ for all $\omega \in\left(T^{*} M\right)_{x}, x \in M, D \in \operatorname{Gr}_{p}(M)$, where $\operatorname{Ann}(W)=\left\{\sigma \in\left(T_{x} M\right)^{*} \mid\right.$ $\langle\sigma, w\rangle=0$ for all $w \in W\}$ is the annihilator of a vector subspace $W \subset T_{x} M$.

ExAmple 3. $A^{[3]}(D)_{\omega}=\operatorname{ker}\left(T_{\omega} \pi_{M}\right)$ for all $\omega \in\left(T^{*} M\right)_{x}, x \in M$, $D \in \underline{\operatorname{Gr}_{p}}(M)$, where $\pi_{M}: T^{*} M \rightarrow M$ is the cotangent bundle projection.

EXAmple 4. $A^{[4]}(D)_{\omega}=\left(T_{\omega} \pi_{M}\right)^{-1}\left(D_{x}\right)$ for all $\omega \in\left(T^{*} M\right)_{x}, x \in M$, $D \in \underline{\mathrm{Gr}_{p}}(M)$.

ExAmple 5. $A^{[5]}(D)_{\omega}=T_{\omega} T^{*} M$ for all $\omega \in\left(T^{*} M\right)_{x}, x \in M$, $D \in \underline{\mathrm{Gr}_{p}}(M)$.

The main result in this paper is the following theorem.

Theorem 1. All $\mathcal{M} f_{m}$-natural operators $A: \mathrm{Gr}_{p} \rightsquigarrow \mathrm{Gr}_{q} T^{*}$ are described in Examples 1-5.

From now on let $x^{1}, \ldots, x^{m}$ be the usual coordinates on $\mathbb{R}^{m}$. Let $D^{p}$ be the integrable $p$-dimensional distribution on $\mathbb{R}^{m}$ spanned by $\partial / \partial x^{1}, \ldots$, $\partial / \partial x^{p}$.

2. First we prove the following proposition.

Proposition 1. All $\mathcal{M} f_{m}$-natural operators $A: \operatorname{Gr}_{p} \rightsquigarrow \operatorname{Gr}_{q} T^{*}$ such that $A(D) \subset V T^{*} M=\operatorname{ker}\left(T \pi_{M}\right)$ for any $D \in \underline{\operatorname{Gr}_{p}}(M)$ are described in Examples 1-3.

LEMma 1. Let $A^{1}, A^{2}: \mathrm{Gr}_{p} \rightsquigarrow \mathrm{Gr}_{q} T^{*}$ be $\mathcal{M} f_{m}$-natural operators such that $A^{1}(D) \subset V T^{*} M$ and $A^{2}(D) \subset V T^{*} M$ for any $D \in \operatorname{Gr}_{p}(M)$. Assume $A^{1}\left(D^{p}\right)_{\theta}=A^{2}\left(D^{p}\right)_{\theta}$, where $\theta \in\left(T^{*} \mathbb{R}^{m}\right)_{0}$ is the zero element. Then $A^{1}(D)_{\omega}=A^{2}(D)_{\omega}$ for any $p$-dimensional distribution $D$ on an $m$-manifold $M$ and any $\omega \in\left(T^{*} M\right)_{x}, x \in M$, i.e. $A^{1}=A^{2}$.

Proof. By the $\mathcal{M} f_{m}$-invariance of $A^{1}$ and $A^{2}$ we may assume that $M=\mathbb{R}^{m}, x=0, D_{0}=D_{0}^{p}$ and $\omega \in\left(T^{*} \mathbb{R}^{m}\right)_{0}$.

We have the standard identification $I_{\omega}: V_{\omega} T^{*} \mathbb{R}^{m}=T_{\omega}\left(T^{*} \mathbb{R}^{m}\right)_{0} \rightarrow$ $\left(T^{*} \mathbb{R}^{m}\right)_{0}=\mathbb{R}^{m *}, \omega \in\left(T^{*} \mathbb{R}^{m}\right)_{0}$. Using the invariance of $A^{i}$ with respect to the homotheties $a_{t}=\left(\frac{1}{t} x^{1}, \ldots, \frac{1}{t} x^{m}\right)$ for $t>0$ we deduce that

$$
I_{t \omega}\left(A^{i}\left(\left(\operatorname{Gr}_{p}\left(\left(a_{t}\right)_{*} D\right)_{t \omega}\right)\right)\right)=I_{\omega}\left(A^{i}(D)_{\omega}\right)
$$


for $i=1$, 2. Letting $t \rightarrow 0$ we deduce that $I_{\omega}\left(A^{i}(D)_{\omega}\right)=I_{\theta}\left(A^{i}\left(D^{p}\right)_{\theta}\right)$ for $i=1,2$. Then $A^{1}(D)_{\omega}=A^{2}(D)_{\omega}$ because of the assumption of the lemma.

Lemma 2. Let $A: \mathrm{Gr}_{p} \rightsquigarrow \mathrm{Gr}_{q} T^{*}$ be an $\mathcal{M} f_{m}$-natural operator such that $A(D) \subset V T^{*} M$ for any $D \in \operatorname{Gr}_{p}(M)$. Then $A\left(D^{p}\right)_{\theta}=A^{[i]}\left(D^{p}\right)_{\theta}$ for some $i=1,2,3$.

Proof. We may assume that $A\left(D^{p}\right)_{\theta} \neq\{0\}$.

If there is a point $v \in A\left(D^{p}\right)_{\theta} \backslash A^{[2]}\left(D^{p}\right)_{\theta}$, then using the invariance of $A$ with respect to linear isomorphisms preserving $D^{p}$ we see that $V_{\theta} T^{*} \mathbb{R}^{m} \backslash A^{[2]}\left(D^{p}\right)_{\theta} \subset A\left(D^{p}\right)_{\theta}$ (if $w \in V_{\theta} T^{*} \mathbb{R}^{m} \backslash A^{[2]}\left(D^{p}\right)_{\theta}$ then there exists a linear isomorphism $\psi$ preserving $D^{p}$ such that $w=T T^{*} \psi(v)$, and thus $w \in A\left(D^{p}\right)_{\theta}$ because of the invariance of $A$ with respect to $\psi$ and $\left.v \in A\left(D^{p}\right)_{\theta}\right)$; consequently, $A\left(D^{p}\right)_{\theta}=A^{[3]}\left(D^{p}\right)_{\theta}$.

So we may assume $A\left(D^{p}\right)_{\theta} \subset A^{[2]}\left(D^{p}\right)_{\theta}$. There is an element $v \in A\left(D^{p}\right)_{\theta}$, $v \neq 0$. Then using the invariance of $A$ with respect to linear isomorphisms preserving $D^{p}$ we see that $A^{[2]}\left(D^{p}\right)_{\theta} \subset A\left(D^{p}\right)_{\theta}$. Thus $A\left(D^{p}\right)_{\theta}=A^{[2]}\left(D^{p}\right)_{\theta}$.

Proof of Proposition 1. Proposition 1 is an immediate consequence of Lemmas 1 and 2.

3. Now, we prove the following proposition.

Proposition 2. Let $A: \mathrm{Gr}_{p} \rightsquigarrow \mathrm{Gr}_{q} T^{*}$ be an $\mathcal{M} f_{m}$-natural operator such that $A(D) \subset A^{[4]}(D)$ for any $D \in \operatorname{Gr}_{p}(M)$ and $A\left(D^{p}\right)_{\theta} \backslash V_{\theta} T^{*} \mathbb{R}^{m} \neq \emptyset$. Then $A=A^{[4]}$.

Lemma 3. Under the assumptions of Proposition 2 we have the inclusion $A\left(D^{p}\right)_{\theta} \supset V_{\theta} T^{*} \mathbb{R}^{m}$.

Proof. There exists $a>0$ such that $A\left(D^{p}\right)_{a d_{0} x^{1}} \backslash V_{a d_{0} x^{1}} T^{*} \mathbb{R}^{m} \neq \emptyset$. Then using the invariance of $A$ with respect to the homotheties $b_{t}=\left(t x^{1}, \ldots, t x^{m}\right)$ for $t>0$ we find that $A\left(D^{p}\right)_{d_{0} x^{1}} \backslash V_{d_{0} x^{1}} T^{*} \mathbb{R}^{m} \neq \emptyset$.

There exist real numbers $a_{1}, \ldots, a_{m}, b_{1}, \ldots, b_{p} \in \mathbb{R}$ such that

$$
\begin{aligned}
Y= & a_{1} \frac{d}{d t}\left[d_{0} x^{1}+t d_{0} x^{1}\right]_{t=0}+\cdots+a_{m} \frac{d}{d t}\left[d_{0} x^{1}+t d_{0} x^{m}\right]_{t=0} \\
& +b_{1} \mathcal{T}^{*}\left(\frac{\partial}{\partial x^{1}}\right)_{d_{0} x^{1}}+\cdots+b_{p} \mathcal{T}^{*}\left(\frac{\partial}{\partial x^{p}}\right)_{d_{0} x^{1}} \in A\left(D^{p}\right)_{d_{0} x^{1}}
\end{aligned}
$$

and $b_{q} \neq 0$ for some $q \in\{1, \ldots, p\}$, where $\mathcal{T}^{*}(X)$ denotes the flow lifting of a vector $X$ on $M$ to $T^{*} M$.

Consider $k \in\{1, \ldots, m\}$. Let $\varphi: \mathbb{R}^{m} \rightarrow \mathbb{R}^{m}$ be a diffeomorphism such that $\varphi^{-1}\left(y^{1}, \ldots, y^{m}\right)=\left(y^{1}+y^{q} y^{k}, y^{2}, \ldots, y^{m}\right)$ on some open neighborhood of $0 \in \mathbb{R}^{m}$. Then $\varphi$ preserves $d_{0} x^{1}$ and $D^{p}$. By standard verification one can 
show that

$$
T T^{*} \varphi(Y)=Y+b_{q} \frac{d}{d t}\left[d_{0} x^{1}+t d_{0} x^{k}\right]_{t=0}+b_{k} \frac{d}{d t}\left[d_{0} x^{1}+t d_{0} x^{q}\right]_{t=0} .
$$

By the invariance of $A$ with respect to $\varphi$ we have $T T^{*} \varphi(Y) \in A\left(D^{p}\right)_{d_{0} x^{1}}$, and so

$$
b_{q} \frac{d}{d t}\left[d_{0} x^{1}+t d_{0} x^{k}\right]_{t=0}+b_{k} \frac{d}{d t}\left[d_{0} x^{1}+t d_{0} x^{q}\right]_{t=0} \in A\left(D^{p}\right)_{d_{0} x^{1}} .
$$

Putting $k=q$ we find $\frac{d}{d t}\left[d_{0} x^{1}+t d_{0} x^{q}\right]_{t=0} \in A\left(D^{p}\right)_{d_{0} x^{1}}$, and hence $\frac{d}{d t}\left[d_{0} x^{1}+t d_{0} x^{k}\right]_{t=0} \in A\left(D^{p}\right)_{d_{0} x^{1}}$. Thus $A\left(D^{p}\right)_{d_{0} x^{1}} \supset V_{d_{0} x^{1}} T^{*} \mathbb{R}^{m}$. Then using the invariance of $A$ with respect to the homotheties $a_{t}=\left(\frac{1}{t} x^{1}, \ldots, \frac{1}{t} x^{m}\right)$ for $t>0$ and next letting $t \rightarrow 0$ we deduce that $A\left(D^{p}\right)_{\theta} \supset V_{\theta} T^{*} \mathbb{R}^{m}$.

Proof of Proposition 2. By Lemma 3 and the assumptions of the proposition, there exists a constant vector field $W=a_{1} \partial / \partial x^{1}+\cdots+a_{p} \partial / \partial x^{p} \neq 0$ on $\mathbb{R}^{m}$ such that $\mathcal{T}^{*}(W)_{\theta} \in A\left(D^{p}\right)_{\theta}$. Using the invariance of $A$ with respect to linear isomorphisms preserving $D^{p}$ we deduce that $\mathcal{T}^{*}\left(\partial / \partial x^{i}\right)_{\theta} \in A\left(D^{p}\right)_{\theta}$ for $i=1, \ldots, p$. Then using Lemma 3 we get $A\left(D^{p}\right)_{\theta}=A^{[4]}\left(D^{p}\right)_{\theta}$. Thus $q=\operatorname{dim}\left(A\left(D^{p}\right)_{\theta}\right)=m+p$. Hence $A(D) \subset A^{[4]}(D)$ is an $(m+p)$-dimensional distribution for any $D \in \operatorname{Gr}_{p}(M)$. Therefore $A(D)=A^{[4]}(D)$ for any $D \in$ $\operatorname{Gr}_{p}(M)$, i.e. $A=A^{[4]}$.

4. Quite similarly to Section 3 we prove the next proposition.

Proposition 3. Let $A: \mathrm{Gr}_{p} \rightsquigarrow \mathrm{Gr}_{q} T^{*}$ be an $\mathcal{M} f_{m}$-natural operator such that $A\left(D^{p}\right)_{\theta} \backslash A^{[4]}\left(D^{p}\right)_{\theta} \neq \emptyset$. Then $A=A^{[5]}$.

LEMMA 4. Under the assumptions of Proposition 4 we have the inclusion $A\left(D^{p}\right)_{\theta} \supset V_{\theta} T^{*} \mathbb{R}^{m}$.

Proof. We modify the proof of Lemma 3 by replacing $p$ by $m$ wherever appropriate.

Proof of Proposition 3. By Lemma 4 and the assumptions of the proposition, there exists a constant vector field $W=a_{1} \partial / \partial x^{1}+\cdots+a_{m} \partial / \partial x^{m}$ with $a_{i} \neq 0$ for some $i=p+1, \ldots, m$ such that $\mathcal{T}^{*}(W)_{\theta} \in A\left(D^{p}\right)_{\theta}$. Then using the invariance of $A$ with respect to the linear isomorphisms preserving $D^{p}$ we deduce that $\mathcal{T}^{*}(U)_{\theta} \in A\left(D^{p}\right)_{\theta}$ for any constant vector field $U=c_{1} \partial / \partial x^{1}+\cdots+c_{m} \partial / \partial x^{m}$ with $c_{j} \neq 0$ for some $j=p+1, \ldots, m$. Hence using Lemma 4 we get $A\left(D^{p}\right)_{\theta}=T_{\theta} T^{*} \mathbb{R}^{m}$. Thus $q=2 m$ and consequently $A=A^{[5]}$.

5. Proof of Theorem 1. Let $A: \operatorname{Gr}_{p} \rightsquigarrow \operatorname{Gr}_{q} T^{*}$ be an $\mathcal{M} f_{m}$-natural operator. By the $\mathcal{M} f_{m}$-invariance of $A$ and Proposition 1 we may assume that $A(D)_{\omega} \backslash V_{\omega} T^{*} \mathbb{R}^{m} \neq \emptyset$ for some $D \in \underline{\mathrm{Gr}_{p}}\left(\mathbb{R}^{m}\right)$ and $\omega \in\left(T^{*} \mathbb{R}^{m}\right)_{0}$. If $p \geq 1$, 
by a density argument we may assume that $\omega \notin \operatorname{Ann}\left(D_{0}\right)$, and then using the invariance of $A$ with respect to linear isomorphisms we may additionally assume that $\omega=d_{0} x^{1}$ and $D_{0}=D_{0}^{p}$. (The case $p=0$ is of of course "equivalent" to the case $p=m$. In these two cases we have only canonical distributions on $T^{*} M$ - as trivial distributions give no information.) Let $v \in$ $A(D)_{d_{0} x^{1}} \backslash V_{d_{0} x^{1}} T^{*} \mathbb{R}^{m}$. Then standardly one can show that there exist a constant vector field $Y^{0} \neq 0$ and a vector field $Y^{1}$ with $Y^{1}(0)=0$ such that $\mathcal{T}^{*}(Y)_{d_{0} x^{1}}=v$, where $Y=Y^{0}+Y^{1}$. There exists a local diffeomorphism $\psi$ such that $j_{0}^{1} \psi=$ id and $\psi_{*} Y=Y^{0}$ near 0 . Using the invariance of $A$ with respect to $\psi$ we may additionally assume $v=\mathcal{T}^{*}\left(Y^{0}\right)_{d_{0} x^{1}}$. Then using the invariance of $A$ with respect to the homotheties $a_{t}=\left(\frac{1}{t} x^{1}, \ldots, \frac{1}{t} x^{m}\right)$ for $t>0$ and letting $t \rightarrow 0$ we have $\mathcal{T}^{*}\left(Y^{0}\right)_{\theta} \in A\left(D^{p}\right)_{\theta} \backslash V_{\theta} T^{*} \mathbb{R}^{m}$. Thus $A\left(D^{p}\right)_{\theta} \backslash V_{\theta} T^{*} \mathbb{R}^{m} \neq \emptyset$.

If $A(D) \subset A^{[4]}(D)$ for any $D \in \underline{\operatorname{Gr}_{p}}(M)$, then $A=A^{[4]}$ because of Proposition 2.

Otherwise we may assume that $A(D)_{\omega} \backslash A^{[4]}(D)_{\omega} \neq \emptyset$ for some $D \in \operatorname{Gr}_{p}\left(\mathbb{R}^{m}\right)$ with $D_{0}=D_{0}^{p}$ and some $\omega \in\left(T^{*} \mathbb{R}^{m}\right)_{0}$. Then taking $v \in$ $A(D) \overline{d_{0} x^{1}} \backslash A^{[4]}(D)_{d_{0} x^{1}}$ we obtain (as above) $\mathcal{T}^{*}\left(Y^{0}\right)_{\theta} \in A\left(D^{p}\right)_{\theta} \backslash A^{[4]}\left(D^{p}\right)_{\theta}$. Thus $A\left(D^{p}\right)_{\theta} \backslash A^{[4]}\left(D^{p}\right)_{\theta} \neq \emptyset$. Finally, $A=A^{[5]}$ because of Proposition 3 .

\section{References}

[1] I. Kolář, P. W. Michor and J. Slovák, Natural Operations in Differential Geometry, Springer, Berlin, 1993.

Institute of Mathematics

Jagiellonian University

Reymonta 4

30-059, Kraków, Poland

E-mail: Wlodzimierz.Mikulski@im.uj.edu.pl

Received 16.10.2007

and in final form 18.1.2008

(1822) 